\title{
An Adapting Mechanism to Manipulate Manual Wristed Laparoscopic Instruments by a Robotic Surgery System
}

\author{
Shahrzad Hanifeh ${ }^{1}$, Alireza Alamdar ${ }^{1}$, Farzam Farahmand ${ }^{1}$, Alireza Mirbagheri \\ ${ }^{1}$ Mechanical Engineering Department, Sharif University of Technology \\ Tehran, Iran \\ ${ }^{2}$ Department of Medical Physics \& Biomedical Engineering, School of Medicine, Tehran University of Medical Sciences \\ Tehran, Iran \\ sh.hbkh@gmail.com; alireza.alamdar@gmail.com; farahmand@sharif.edu; a-mirbagheri@tums.ac.ir.com
}

\begin{abstract}
Sina is a robotic telesurgery system that accepts and works with conventional laparoscopic tools at its endeffector. This paper describes the design and analysis of an adapting mechanism for Sina, to enable manipulating a manual wristed laparoscopic instrument, i.e., Laparo-Angle by the system. After identification of the kinematics and dynamics characteristics of Laparo-Angle experimentally, the design considerations of the adapting mechanism, including compatibility with the instrument (at one side) and the host robotic arm (at the other side), required kinematics and dynamics features, ease of tool replacement, etc., were studied and a conceptual design, based on the Agile Eye mechanism, was proposed. The forward and inverse kinematics analysis of the proposed mechanism revealed that it is capable of providing the desired workspace while meeting the design constraints. The workspace of the mechanism was a cone with an apex angle of 35 degrees, in which both the isotropy and manipulability indices were satisfying. The condition number and the Jacobean determinant of the mechanism within its workspace were lower than 1.4 and higher than 1, respectively. In general, the proposed mechanism was found to could satisfy the design requirements and constrains.
\end{abstract}

Keywords: wristed instrument, Sina surgical robotic system, activation mechanism

\section{Introduction}

Compared to open surgery, Minimally Invasive Surgery (MIS) techniques, such as laparoscopic surgery, bring a large number of benefits to the patient, including less pain, blood loss, and infection, as well as a faster recovery. However, because of performing the operation through small incisions, it is much more challenging for the surgeons. This is partly due to the fact that, the surgeon loses direct 3D vision from the surgery site. But, perhaps more importantly, due to the fact that he has to work with long narrow instruments with limited degrees of freedom (DOFs). Such tools not only disturb the surgeon's sense of touch, but also limit his manoeuvres during surgery, resulting in more exhausting and longer operation times [1].

Wristed laparascopic instruments, such as Laparo-Angle [2], have a further wrist joint near their distal tip, which enables the distal tip bends in any direction and turns at any angle. Adding the wrist, has been reported to bring back the lost DOFs and increases the maneuverability of the system to a great extent [3-6]. The importance of the wrist joint is particularly critical in difficult surgeries such as prostatectomy in which a high manoeuvrabitiy is required to avoid cutting the nerves in the surgical site [7]. With such an important role, thus, it is not unexpected that having wristed instruments, e.g., EndoWrist, is considered as an essential feature for modern surgical robotic systems, e.g., davinci ${ }^{\circledR}$ (Intuitive Surgical, CA, USA).

Sina, is a robotic telesurgery system, being developed in the Research Centre for Biomedical Technologies and Robotic, Tehran, Iran. It has been designed to have a modular and configurable structure, improving its capabilities and reducing its maintenance and operations costs. In particular, the system can accept and work with conventional laparoscopic tools at its endeffector, eliminating the need to exclusively designed surgical tools that increase the cost of surgery substantially [8-9]. This paper reports the design and analysis of an adapting mechanism, which is integrated within the Sina robotic surgery system, to enable the system working with a manual wristed laparoscopic instrument, i.e., LaparoAngle [2]. After identification of the instrument's kinematics and dynamics, the design considerations of the adapting 
mechanism are investigated. Then, based on the design requirements and constraints, an Agile Eye mechanism is proposed and its forward and inverse kinematics analysed to examine if the design considerations are met.

\section{Identification of Laparo-Angle Instrument}

Laparo-Angle wristed laparoscopic instrument [2] has 4 DOFs: two wrists - pitch and yaw, a distal roll, and a grasp. The distal tip of the instrument can be bent up to 90 degrees in each direction. The distal roll of the tip, on the other hand, enables the surgeon to roll the distal tip $\pm 180^{\circ}$ in place, i.e., around the bended axis of distal end (not the tool main rod). The surgeon can impose pitch and yaw movements by bending the tool handle. There are also a rotation knob and a finger loop on the tool handle for creating distal roll and grasp, respectively.

In order to design an effective actuation mechanism for manipulating Laparo-Angle, it is required that the tool's kinematics and dynamics behaviour are fully identified. In particular, the ranges of motion of the different DOFs and their relationship with the relevant motions at the knobs of the handle are needed in order to define the workspace of the adapting mechanism. A series of tests were performed on the tool to reveal such information. The kinematics tests showed that the bending of tool handle is magnified at the distal tip, so that a $30^{\circ}$ bent at the tool handle results in an $80^{\circ}$ bent at the distal tip. Equation (1) describes the derived linear relationship between these two angles in degrees, which was used later to define the workspace the adapting mechanism.

$$
\theta_{\text {Handle }}=0.4077 \theta_{\text {Wrist }}-0.9885
$$

Aseries of tests were also performed on the surgical tool to determine the forces that are required for actuation of each of the wrist, roll, and grasp DOFs. This information were later used to evaluate the dynamics efficacy of the designed mechanism and select the motors for the actuation unit. It was hypothesized that the required torque for bending the handle comes from three sources: 1) the force exerted from the tissue to the tip of the tool that is then translated to the handle, 2) the friction at the wrist mechanism of the tool, and 3) the gravity. Taking into account all three sources, the maximum resultant torque for the wrist motion was $1.184 \mathrm{~N} . \mathrm{m}$. Also, our measurements for the torques required for the distal roll and the force required for the grasp motion of the tool revealed 0.24 N.m and $4.68 \mathrm{~N}$ respectively.

\section{Conceptual Design}

The design problem concerned with a mechanism that could be integrated into the distal arm of the Sina surgical robot and fully replace the surgeon's hand in holding and manipulating the wristed laparoscopic instrument. In other words, the mechanism should provide all the motions that a surgeon creates when working with instrument's ergonomic handle. To achieve an effective solution for this design problem, we first investigated the design requirements and considerations in detail.

\subsection{Design Considerations}

The design considerations for the adapting mechanism were categorized as follows:

Kinematics Considerations (Workspace): The adapting mechanism should be able to obtain the full workspace of the Laparo-Angle which is $\pm 90^{\circ}$ for pitch and yaw, a continuous roll, and a full range grasp. We defined a simple test to measure the handle bent required for reaching the full range bending on the distal tip. The results showed that the handle travels inside a cone with an apex angle of $35^{\circ}$.

Compatibility with the Tool: The handle and the rod of Laparo-Angle are connected with a specially designed plastic ball and socket joint. There is a spherical sector at the end of the proximal bending section around which the mating spherical sector on the tool handle pivot. So, the tool handle movements can be approximated by pure rotation around a fixed and remote centre of motion (RCM) on the tool rod, i.e., the centre of the spherical sectors. Therefore, the adapting mechanism should provide a 2DOF spherical movement around tool's RCM.

Ease of Tool Replacement: A main idea in the design of Sina surgical robot is to utilize low-cost disposable commercial instruments in robotic surgery. While the adapting mechanism is integrated with the host robotic arm, the tool is changed in each surgery, or even several times during a single surgery due to the need for different kinds of tool, e.g., grasper, scissor, etc. Therefore, it is important that the tool could be easily attached/detached to/from the mechanism. 
Dynamic Considerations: The adapting mechanism should provide sufficiently high forces for manipulation of the wristed instrument throughout the workspace. Also, the mechanism should be stiff and strong enough to withstand the internal and external mechanical forces. It is also important to avoid approaching the singular points of the mechanism as they might need very large forces.

Geometric considerations: The adapting mechanism should be easily integrate with Sina surgical system. It should also be as light-weight and small as possible. The collision between the mechanism and the host robotic arm, mechanism and the tool, and the mechanism's internal links should be avoided. To make sure that no such collision can take part, we built a detailed model of the designed mechanism in SolidWorks and carefully investigated the different modes of collision. Then, we proposed an optimization process to find a configuration in which no collision occurs.

\subsection{Design of Adapting Mechanism}

The adapting mechanism should provide the full range of motion for each DOF of the Laparo-Angle instrument. The DOFs of the instrument include two wrists, one distal roll and one grasp. The wrist motion is created by a 2D spherical mechanism. The distal roll is achieved by rotating the tool's rod. And finally, the grasp motion is provided by moving the instrument's finger loop using a linear actuator.

For the wrist motion we decided to use a parallel mechanism, considering the fact it has several advantages over a serial one, including higher accuracy, more stiffness, lower mass and inertia, and more compactness [10]. However, the desired parallel mechanism should provide the required workspace with a small size and minimum number of motors and links. After a thorough study on various types of parallel spherical mechanisms, we came to the idea of a 2D agile eye robot. Agile eye is a parallel spherical mechanism that provides 2 pure rotations around its centre [11]. So, if we install the instrument in such a way that the pivot point (RCM) of the instrument coincide with the agile eye centre, we can bend the tool handle and create the wrist motion with the mechanism. The final design for the wrist actuator of the mechanism is shown in figure 1. We modified the original agile eye structure in order to make it compatible with the design requirements. The wrist actuator includes two motors and four links, that all links are attached to each other by revolute joints.

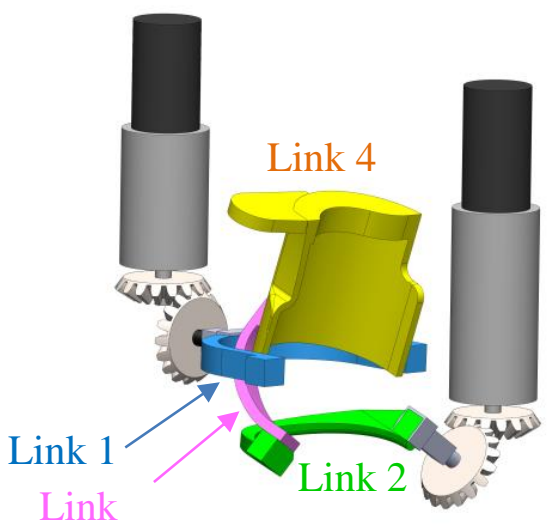

Fig. 1: The wrist actuator of the adapting mechanism.

Considering figure 1, two kinematic chains are distinguished for the mechanism. In the first chain, the first motor rotates link 1 that is attached to link 4 at the end-effector of the wrist actuator (Figure 2-left). The second chain consists of links 2 to 4, which are attached together consecutively, and the second motor that rotates link 2 (Figure 2-right). In general, the two motors should be mounted perpendicularly. The end-effector of the mechanism (link 4) experiences a pure rotation around the RCM point, which is the intersection of the two motors' axes. We positioned the two motors of the wrist actuator in vertical alignment, to avoid collision with the host system, and used a set of bevel gears for each motor to transfer power from motors to the links (figure 1).

The final conceptual model of the adapting mechanism is shown in figure 3 . To provide the roll motion, we attached a small spur gear to the instrument's rod. There is also a mating pinion assembled on the base of the mechanism. After placing the instrument inside the robot, the two gears engage. The roll motor rotates the pinion; the pinion rotates the gear and therefore the rod. By rotating the rod, the distal roll of the tool occurs. 
The finger loop has a mixed rotation/translation motion. Therefore, we used a float linear actuation for grasping. We used a unit of motor and ball screw to create linear motion (figure 3). One end of the actuation unit is pivoted to the mechanism base. Before handing the tool to the robot, the other end is free. After placing the tool inside the mechanism, we can manually position the free end in handle's finger loop.
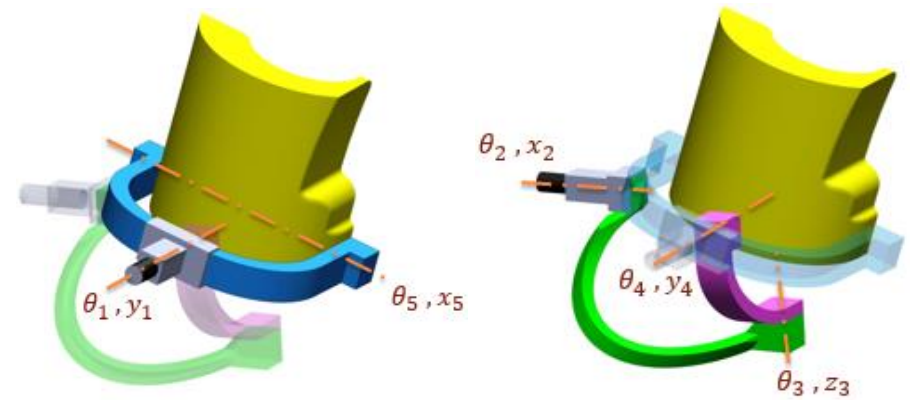

Fig. 2: The first (left) and second (right) kinematic chains of wrist actuator.
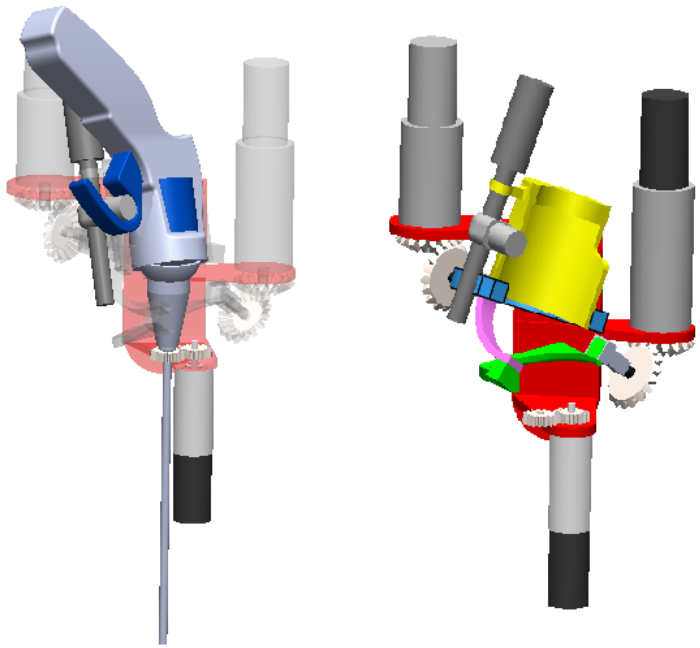

Fig. 3: The adapting mechanism with (left) and without (right) the instrument.

\section{Kinematics Analysis}

In this section, we provide the kinematics equations for the wrist actuator. These equations are then used to ensure that the designed mechanism can effectively cover the desired workspace. The forward kinematics gives the position of the endeffector for a given angular position of the pitch and yaw motors. We use these equations to obtain the workspace and to determine the singular positions of the mechanism. Furthermore, in order to accurately control the instrument's DOFs, we need to have access to the inverse kinematics solution of the mechanism. We use equation 1 to transform the wrist angle into the handle's bending, and then obtain the motors' angular position, corresponding to the handle orientation, using inverse kinematics. We also derive the Jacobean matrix of the mechanism in order to calculate its kinematic manipulability and isotropy for optimization purposes. The results of the forward and inverse kinematics, as well as the Jacobean matrix, are used to check whether a specific design of the mechanism fulfils the workspace requirement.

\subsection{Forward Kinematics}

To analyze the forward kinematics of the system, we first assign a coordinate system to each link. Then we calculate the transformation matrix between different links of the mechanism. We name the global coordinate system as 0 , and the coordinate systems on links 1 to 4 as 1,2,3, and 4, respectively. The joint parameters of the hinges of the mechanism are shown in figure 2 and figure 3 . Matrix ${ }_{4}^{0} R$ determines the position and orientation of the end-effector (link 4 ) with respect to 
the global coordinate system. We derive this matrix from both kinematic chains (equations (2) and (3) for first and (4) and (5) for second kinematic chain).

$$
\begin{gathered}
{ }_{4}^{0} R={ }_{1}^{0} R \times{ }_{4}^{1} R \\
{ }_{4}^{0} R=\operatorname{Rot}\left(y_{1}, \theta_{1}\right) \times \operatorname{Rot}\left(x_{5}, \theta_{5}\right) \\
{ }_{4}^{0} R={ }_{2}^{0} R \times{ }_{3}^{2} R \times{ }_{4}^{3} R \\
{ }_{4}^{0} R=\operatorname{Rot}\left(x_{2}, \theta_{2}\right) \times \operatorname{Rot}\left(z_{3}, \theta_{3}\right) \times \operatorname{Rot}\left(y_{4}, \theta_{4}\right)
\end{gathered}
$$

In forward kinematics, the known parameters are the motor joint angles $\left(\theta_{1}\right.$ and $\left.\theta_{2}\right)$ and the unknown parameters are the location of the end-effector $\left({ }_{4}^{0} R\right)$, as well as the mechanism configuration $\left(\theta_{3}, \theta_{4}\right.$ and $\left.\theta_{5}\right)$. The right-hand-side of equations 3 and 5 are equal, which results in 9 equations from which 3 are independent. By solving these equations, we determine $\theta_{3}, \theta_{4}$ and $\theta_{5}$ and subsequently the end-effector transformation matrix ${ }_{4}^{0} R$. The third column of this matrix corresponds to the orientation of the tool.

\subsection{Inverse Kinematics}

In inverse kinematics, the location of the end-effector of the mechanism in the global coordinate system (tool orientation) is given and the joint parameters $\left(\theta_{1}\right.$ to $\left.\theta_{5}\right)$ should be calculated. Equation (6) describes ${ }_{4}^{0} R$ in terms of $\theta_{1}$ and $\theta_{5}$. The last column of this equation is the tool orientation which is known, enabling to calculate $\theta_{1}$ to $\theta_{5}$. Having $\theta_{1}$ and $\theta_{5}$, we can then calculate ${ }_{4}^{0} R$ and find $\theta_{2}$ to $\theta_{4}$ from equation (7). In equations (6), (7) and (10), $s_{i}$ and $c_{i}$ are the short from of $\sin \theta_{i}$ and $\cos \theta_{i}$, respectively.

$$
\begin{gathered}
{ }_{4}^{0} R=\left[\begin{array}{ccc}
c_{1} & s_{1} s_{5} & c_{5} s_{1} \\
0 & c_{5} & -s_{5} \\
-s_{1} & c_{1} s_{5} & c_{1} c_{5}
\end{array}\right] \\
{ }_{4}^{0} R=\left[\begin{array}{ccc}
c_{3} c_{4} & -s_{3} & c_{3} s_{4} \\
s_{2} s_{4}+c_{2} c_{4} s_{3} & c_{2} c_{3} & c_{2} s_{3} s_{4}-c_{4} s_{2} \\
c_{4} s_{2} s_{3}-c_{2} s_{4} & c_{3} s_{2} & c_{2} c_{4}+s_{2} s_{3} s_{4}
\end{array}\right]
\end{gathered}
$$

\subsection{Jacobean Matrix}

The Jacobean matrix shows the relationship between the end-effector's angular velocity and the motor speeds $\left(\dot{\theta}_{1}\right.$ and $\dot{\theta}_{2}$ ). We use equations (8) and (9) to calculate the angular velocity of the consecutive links.

$$
\begin{gathered}
\omega={ }^{0} J \dot{\theta} \\
{ }^{i+1} \omega_{i+1}={ }^{i+1} R{ }^{i} \omega_{i}+\dot{\theta}_{i+1}{ }^{i+1} \hat{z}_{i+1}
\end{gathered}
$$

where ${ }^{i} \omega_{i}$ is the angular velocity of ith link described in ith coordinate system. The Jacobean matrix is determined as follows:

$$
0=\frac{1}{c_{4}}\left[\begin{array}{cc}
c_{1} s_{4} s_{5} & c_{1} c_{3} \\
1 & 0 \\
-s_{1} s_{4} s_{5} & -s_{1} c_{3}
\end{array}\right]
$$


In order to calculate the performance indices of the mechanism, we need the Jacobean determinant. Since the matrix is non-square, we can calculate the Jacobean of $J^{T} J$ matrix instead.

\section{Results and Discussion}

We solved the forward and inverse kinematics of the wrist actuator of the designed mechanism. In general, the mechanism was found to have 12 singular positions which are listed in table 1 . The optimized mechanism was found to cover the whole workspace- a cone with apex angle of $35^{\circ}$ - without being trapped in a singular situation. Figure 5 shows the isotropy and manipulability indices of the mechanism throughout its workspace. From figure 5-left, the Jacobean determinant remains larger than 1 inside the entire workspace which indicates that the mechanism is far from its singularities. From figure 5-right, the maximum value of the condition number is 1.4 which reflects a well isotropic mechanism. Therefore, reasonably acceptable levels of kinematic manipulability and kinematic isotropy are achieved within the desired workspace.

Table 1: Singular positions of the wrist actuator.

\begin{tabular}{|c|c|c|c|c|c|}
\hline & $\theta_{1}$ & $\theta_{2}$ & $\theta_{3}$ & $\theta_{4}$ & $\theta_{5}$ \\
\hline 1 & 90 & arbitrary & 90 & $90+\theta 2$ & 270 \\
\hline 2 & 270 & arbitrary & 90 & $270+\theta 2$ & 90 \\
\hline 3 & 90 & arbitrary & 270 & $90-\theta 2$ & 90 \\
\hline 4 & 270 & arbitrary & 270 & $90-\theta 2$ & 270 \\
\hline 5 & arbitrary & 90 & $-\theta 1$ & 0 & 90 \\
\hline 6 & arbitrary & 90 & $180-\theta 1$ & 180 & 270 \\
\hline 7 & arbitrary & 270 & $180+\theta 1$ & 180 & 90 \\
\hline 8 & arbitrary & 270 & $\theta 1$ & 0 & 270 \\
\hline 9 & 90 & 0 & arbitrary & 90 & $-\theta 3$ \\
\hline 10 & 270 & 180 & arbitrary & 90 & $180-\theta 3$ \\
\hline 11 & 90 & 180 & arbitrary & 270 & $180+\theta 3$ \\
\hline 12 & 270 & 0 & arbitrary & 270 & $\theta 3$ \\
\hline
\end{tabular}
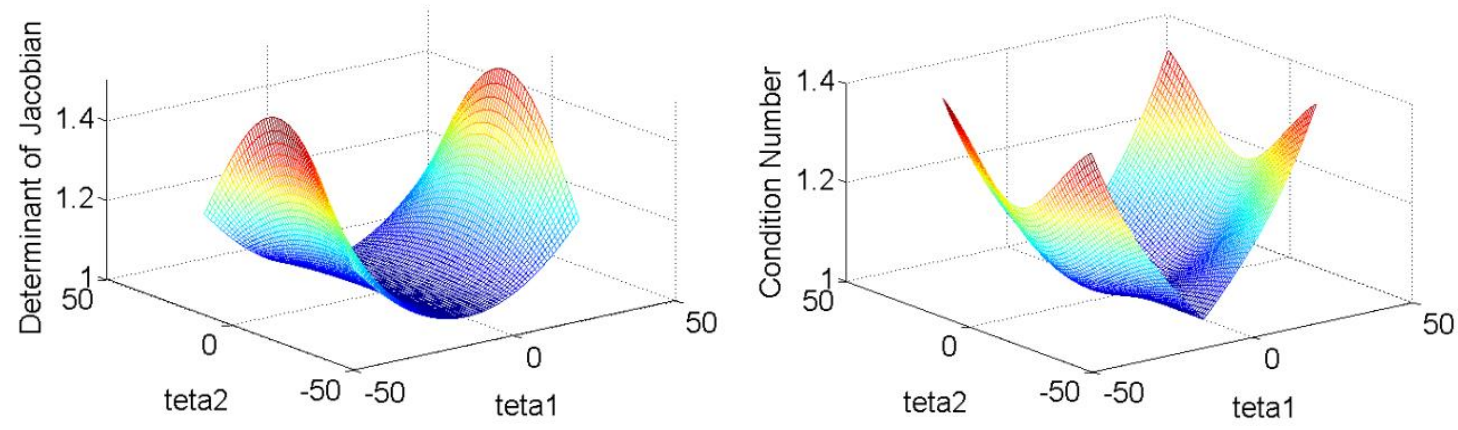

Fig. 5: Isotropy (left) and manipulability (right) indices of the wrist actuator throughout the desired workspace.

In order to optimize the dimensions of the designed mechanism against any probable collision, a 3D model of the system was created in SolidWorks. The system consisted of the Laparo-Angle instrument, the adapting mechanism, and the Sina robot. We investigated the different forms of collision in SolidWorks, created an algebraic model for each form and programmed it in Matlab software. Then we used them as geometric constraints in our optimization algorithm. We also took into account other design considerations such as ease of tool replacement and compatibility with the tool to finalize the design.

The final optimal model of the mechanism is shown in figure 6 . A connecting plate between the Sina and the adapting mechanism acts as the base for the latter. We placed the pitch, yaw and roll motors on the connecting plate and the insert 
motor on the mechanism's end-effector. When Laparo-Angle is placed in the mechanism, the end-effector holds the instrument's handle and the trocar holder, at the bottom of the Sina robot, holds the rod. As a result, the rod would be aligned with the tool's insert direction and moving the link 4 would result in the handle's bent and consequently the wrist's motion at the distal end of the instrument. On the other hand, by placing the tool, the pinion on the roll motor and the mating gear on the instrument's rod get engaged automatically which activates the roll motion. Finally, after placing the tool, the linear actuator can be manually engaged with the finger loop to provide the grasp motion.

\section{Conclusion}

In this paper, we proposed an adapting mechanism for manipulation of the Laparo-Angle wristed instrument by Sina robotic surgery system. The kinematic analysis of the wrist actuator showed that the mechanism is capable of providing the desired workspace while satisfying the design constrains for the kinematic manipulability and isotropy. The proposed mechanism also fulfils other design requirements that were considered for the system including compatibility with the tool and ease of tool replacement. A prototype of the designed mechanism has been fabricated and is now under experimental examination.

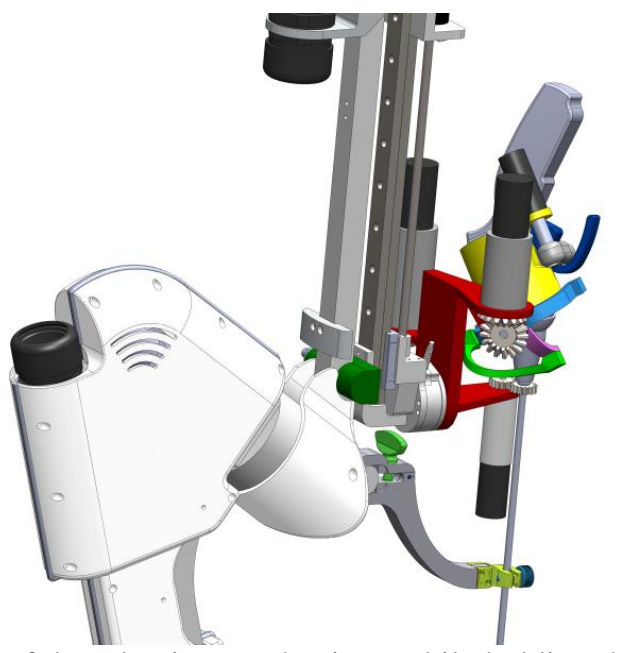

Fig. 6: The final model of the adapting mechanism, while holding the instrument, integrated with Sina robot.

\section{References}

[1] V. Gupta, et al., "Forces in surgical tools: Comparison between laparoscopic and surgical forceps," in Proceedings of IEEE Int. Conf. Engineering in Medicine and Biology Society, Amsterdam, Neth, vol. 1, pp. 223-224, 1996.

[2] Cambridge Endo Autonomy, Laparo-Angle Articulating Instrument Technology: Enabling Visualization, Access \& Control in Single Port Laparoscopy. [Online], Available: http://www.cambridgeendo.com/technology

[3] Y. Kobayashi, et al, "Small occupancy robotic mechanisms for endoscopic surgery," Medical Image Computing and Computer-Assisted Intervention-MICCAI 2002, Tokyo, Japan, pp. 75-82, 2002.

[4] K. Harada, et al, "Micro manipulators for intrauterine fetal surgery in an open MRI," in Proceedings IEEE Int. Conf. Robotics and Automation, Barcelona, Spain, pp. 502-507, 2002.

[5] H. Yamashita, et al, "Development of endoscopic forceps manipulator using multi-slider linkage mechanisms," $J$. Japan Society of Computer Aided Surgery, vol. 7, pp. 201-4, 2005.

[6] J. Arata, et al, "Development of a dexterous minimally-invasive surgical system with augmented force feedback capability," in Proceedings of IEEE Int. Conf. Intelligent Robots and Systems, Edmonton, AB, Canada, pp. 3207$3212,2005$.

[7] A. Lorincz, et al, "Totally minimally invasive robot-assisted unstentedpyeloplasty using the Zeus Microwrist Surgical System: An animal study," J.Pediatric Surgery, vol. 40, pp. 418-422, 2005.

[8] Intuitive Surgical Inc., The da Vinci ${ }^{\circledR}$ Surgical System. [Online]. Available: http://www.davincisurgery.com/davinci-surgery/da-vinci-surgical-system/ 
[9] E. J. Hanly and M. A. Talamini, "Robotic abdominal surgery," J. The American journal of surgery, vol. 188, pp. 1926, 2004.

[10] Y. D. Patel, et al, "Parallel Manipulators Applications-A Survey," J. Modern Mechanical Engineering, pp. 57-64, 2012.

[11] C. M. Gosselin, et al, "The agile eye: a high performance three-degree-of-freedom camera-orienting device," in Proceedings of IEEE Int. Conf. Robotics and Automation, San Diego, CA, United States, vol.1, pp. 781-786, 1994. 